\title{
EXTENSIONS OF DERIVATIONS
}

\section{MICHAEL BARR AND MAX-ALBERT KNUS}

Abstract. We show that for a class of algebras including separable algebras one can extend derivations of the center to derivations of the algebra.

The following theorem was proved in the special cases that $C$ is a field by Hochschild [Ho] and $C$ is a semilocal ring by Roy and Sridharan $[\mathbf{R}, \mathbf{S}]$ [and for any $C$ in $[\mathrm{Kn}]]$. It is also a trivial consequence of a more general result proved by a short cohomological argument.

THEOREM 1. Let $A$ be an algebra separable over its center $C$ and $M$ be an $A \otimes_{C} A^{\mathrm{op}-m o d u l e . ~ T h e n ~ a n y ~ d e r i v a t i o n ~} d: C \rightarrow M^{A}$ extends to a derivation $\tilde{d}: A \rightarrow M$.

Since an algebra separable over its center $C$ is $C$-projective [A, G, p. 379], Theorem 1 follows from

Theorem 2. Let $A$ be a C-algebra, projective over $C$, of Hochschild dimension one and let $M$ be an $A \otimes_{C} A^{\mathrm{op}}-$ module. Then any derivation $d: C \rightarrow M^{A}$ extends to a derivation $\tilde{d}: A \rightarrow M$.

Proof. Let $B$ be the split extension of $A$ by $M$. That is, $B$ is the additive group $A \oplus M$ with $(a, m)\left(a^{\prime}, m^{\prime}\right)=\left(a a^{\prime}, a m^{\prime}+m a^{\prime}\right)$. If we let $C$ operate on $B$ by $c(a, m)=(c a, c m+d c \cdot a)=(a, m) c$, then $B$ is a $C$-algebra and the projection of $B$ to $A$ is a $C$-algebra homomorphism. It is also $C$-linearly split since $A$ is $C$-projective. Thus the extension is an element of $H_{C}^{2}(A, M)$ which is zero by hypothesis. This means that there is a $C$-algebra splitting of $B \rightarrow A$, the second coordinate of which is easily seen to be a derivation extending $d$.

Corollary 1. Let $A, C, M$ be as above and $A_{0}$ be a separable $C$-subalgebra of $A$. Then any derivation $d: A_{0} \rightarrow M$ which takes $C$ to $M^{\boldsymbol{A}}$ can be extended to a derivation $\tilde{d}: A \rightarrow M$.

Proof. First restrict to $C$, then extend to $A$. The difference, on $A_{0}$, is $C$-linear and hence inner.

Received by the editors June 22, 1970.

AMS 1970 subject classifications. Primary 16A16, 16A62, 16A72, 18H15.

Key words and phrases. Separable algebras, Azumaya algebras, algebras of Hochschild dimension one, derivations, extension of derivations. 
Corollary 2. Let $A$ be a R-algebra separable over its center $C$ and

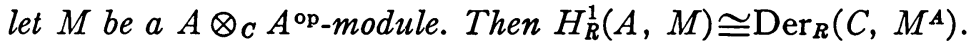

Proof. It is evident that any derivation of $A$ to $M$ restricts to a derivation $C \rightarrow M^{A}$.

\section{REFERENCES}

[Ho]. G. Hochschild, Restricted Lie algebras and simple associative algebras of characteristic p, Trans. Amer. Math. Soc. 80 (1955), 135-147. MR 17, 282.

[R, S]. A. Roy and R. Sridharan, Derivations in Azumaya algebras, J. Math. Kyoto Univ. 7 (1967), 161-167. MR 36 \#5125.

[Kn]. M. A. Knus, Sur le théorème de Skolem-Noether et sur les dérivations des algèbres d'Azumaya, C. R. Acad. Sci. Paris 270 (1970), 637-639.

[A, G]. M. Auslander and O. Goldman, The Brauer group of a commutative ring, Trans. Amer. Math. Soc. 97 (1960), 367-409. MR 22 \#12130.

Forschungsinstitut für Mathematik, Eidgenössiche Technische HochSCHULE, ZÜRICH, SWITZERLAND 